\title{
An Optimized Resilient Advance Bandwidth Scheduling for Media Delivery Services
}

\author{
Maryam Barshan ${ }^{(凶)}$, Hendrik Moens, Bruno Volckaert, and Filip De Turck \\ Department of Information Technology, Ghent University - IMEC, \\ Technologiepark-Zwijnaarde 15, 9052 Gent, Belgium \\ maryam. barshan@intec. ugent. be
}

\begin{abstract}
In IP-based media delivery services, we often deal with predictable network load and traffic, making it beneficial to use advance reservations even when network failure occurs. In such a network, to offer reliable reservations, fault-tolerance related features should be incorporated in the advance reservation system. In this paper, we propose an optimized protection mechanism in which backup paths are selected in advance to protect the transfers when any failure happens in the network. Using a shared backup path protection, the proposed approach minimizes the backup capacity of the requests while guaranteeing $100 \%$ single link failure recovery. We have evaluated the quality and complexity of our proposed solution and the impact of different percentages of backup demands and timeslot sizes have been investigated in depth. The presented approach has been compared to our previously-designed algorithm as a baseline. Our simulation results reveal a noticeable improvement in request acceptance rate, up to $9.2 \%$. Moreover, with fine-grained timeslot sizes and under limited network capacity, the time complexity of the proposed solution is up to $14 \%$ lower.
\end{abstract}

Keywords: Advance bandwidth reservation · Resilient reservation • Fixed timeslot size $\cdot$ Media delivery service

\section{Introduction}

Currently, in the media-centric industries, the distribution of media content is generally performed by either people transporting the content on a physical storage media or over dedicated point-to-point high-speed optical links. However, these are highly inefficient and costly methods. In order to support decentralized collaboration, reduce capital expenditures and increase network resource utilization, media related environments tend to switch to the cost-effective IPbased WAN approaches. Deploying a shared IP-based WAN solution enables the existing media content owners and their collaborators to work together in a cost effective way, while new actors can more easily find new collaboration opportunities, thus fostering the whole industry's further growth.

As media-centric networks usually offer predictable network traffic, this knowledge of future transmissions can be exploited to use advance reservation

(C) The Author(s) 2017

D. Tuncer et al. (Eds.): AIMS 2017, LNCS 10356, pp. 79-93, 2017.

DOI: $10.1007 / 978-3-319-60774-0 \_6$ 
(AR) services. This makes it easier to offer guarantees in advance, improves the number of admitted requests and increases network utilization. In AR techniques, users submit requests for future data transfers, generally encompassing a start time in the future, a deadline, and total data transfer size or rate. To allocate the necessary resources (more specifically network bandwidth), a scheduling algorithm is needed to ensure that all admitted requests finish before their specified deadline, while admitting as many requests as possible. Clearly, AR has advantages for next generation media related networks: it allows network operators to better plan resource usage, leading to greatly increased resource utilization and guaranteed Quality of Service (QoS).

Reliability of the transport is also of crucial importance in the digital-centric media transfer process, when different media actors are geographically located far apart. Therefore, strategies to deal with network dynamics such as failures should be defined to enable reliable transmission of accepted requests without any loss in QoS upon occurrence of a failure. For example, in media production networks, meeting transfers' deadlines is of crucial importance. Consider a live show or a news program which is broadcasted everyday at a specific time. Clearly, even slight delays in transfer of pre-production contents are intolerable in such a setting.

Media-centric networks impose requirements not supported by existing AR scheduling techniques, such as different types of video or audio transfers, flexible or unspecified start or end times, strict deadlines, interdependent requests, reliability, etc. Addressing these unexplored aspects was the main focus of our previous contributions. First optimal and near optimal AR scheduling algorithms, customized for media production networks, have been proposed [1]. To offer reliable reservations, we have further presented the resilient version of our approach based on a protection mechanism to improve the reliability of the AR systems [2]. The proposed scheme is capable of covering single link failures using pre-reserved disjoint backup paths. Additionally, the resilient solution improves the scheme's availability compared to the non-resilient approach.

Continued research has shown us that the resilient bandwidth allocation algorithm, in [2], can be further improved and this is the main contribution of the work presented in this paper. This algorithm is optimized to reduce network reservation waste by proposing a more efficient solution to finding an optimal allocation of bandwidth for each file transfer request. We have made a tradeoff between the complexity and performance of the resilient bandwidth allocation algorithm for file-based transfers. This results in better network utilization and consequently higher request admittance ratio.

The remainder of this paper is structured as follows. In Sect.2, we discuss the related work. Section 3, provides brief information about the media delivery services and elaborates on the resilient AR approach. The proposed solution to improve the performance of resilient AR scheduling approach is described in Sect. 4. Designed algorithms are explained in Sect. 5. Section 6 provides simulation results and finally, Sect. 7 concludes the paper. 


\section{Related Work}

The authors in [3] survey the AR algorithms, mostly focusing on WavelengthDivision Multiplexing (WDM) networks, and provide a taxonomy for classifying these algorithms. Advance reservation requests can be classified in 4 individual categories, which are also valid for different types of requests in media related networks. However, based on this survey, only two works offer variable-bandwidth reservation in their scheduling process $[4,5]$. While both approaches consider a fixed start time for the requests, all four classes for requests with specified or unspecified time and duration are supported in our work. Current research on AR scheduling mostly focuses on rescheduling [6-8], multi-domain reservations [9], and real-life deployments [10-13]. Nevertheless, reliability and fault tolerance properties have not been investigated.

Resilient AR systems can be deployed either through restoration or protection failure recovery mechanisms [14]. In protection approaches, backup resources are reserved in advance before any failure happens in the network, while in case of restoration backup resources are found upon failure detection. The former results in more resource consumption but the recovery time is quite fast. In [15], the authors propose a restoration technique to deal with link failures. In their work, the active requests and the scheduled requests for the future which are affected by a failure are restored. In [16,17], optimal ILP-based solutions were proposed to provide shared and dedicated path protection. Authors in $[18,19]$ also provide resiliency through shared path protection. Since meeting strict deadlines and QoS requirements is of great importance in our approach, we have made use of protection mechanisms.

The work detailed in this paper presents a significant optimization to our previous works on bandwidth reservation approaches [1,2]. In [1], we proposed a theoretical Integer Linear Programming (ILP) based model and heuristic algorithms for advance bandwidth reservation with no support for failure recovery. In [2], the media production reservation system is enhanced by following a protection mechanism and provisioning backup reservations for each request. This resilient solution guarantees $100 \%$ recovery against any single link failure. While this approach strives to minimize the needed bandwidth for the backup paths and determination of allocations is fast it does not fully utilize the network capacity. This work aims at optimizing the resilient solution proposed in [2] and improving the request admittance ratio by reducing wasted network capacity and thus improving network utilization.

\section{Background}

We briefly explain about the specific properties of media delivery networks and provide a summery of the resilient advance bandwidth reservation approach.

\subsection{Media Delivery Networks}

In the digital-centric media related industries, various actors are connected to a shared wide area network to build a collaboration over IP media contents. 
This shared network supports the exchange of different media contents, e.g. raw and encoded video and audio files and streaming transfers. We refer to each transfer as a request. A request can have a fixed or unspecified start and end times. Media delivery requests, supported in our work, are of 4 different classes: independent streaming requests, independent file transfers, dependent streams and dependent file based transfers. The requests of independent type can be started at the specified start time but dependent requests have to wait until the requests upon which they are dependent have finished. Dependency among different transfers implies that either all or none of the interdependent requests must be admitted. We refer to a set of interdependent requests as a scenario.

We assume that volume for file-based requests, and duration for streaming requests duration must be specified. The allocated bandwidth for the streams must be equal to their required bandwidth demand, from the start time to the end time, because their demand is fixed. However, for file-based requests, the volume of file is the determinative factor. The file can be transferred whenever possible from the time the file is ready to be transferred till its deadline. The residual demand of file-based transfers is modified whenever a part of the file is transferred.

\subsection{Resilient AR Scheduling}

In order to have a quick response to sudden changes such as failures in the network, we use a protection mechanism which finds backup paths for connections in advance, before the occurrence of any failure to ensure there is enough capacity left when failures occur. The objective is to minimize the resource usage by the protection paths while full recovery is guaranteed against any single link failure. In this scheme, first the primary paths for a given request are determined using an advance reservation algorithm which we presented in [1]. Then disjoint backup paths are found corresponding to these primary paths [2]. Note that in the proposed schemes, the user can indicate the amount of required backup for each request. This way, the higher priority requests can be fully protected while the ones with lower priority can remain partially protected or unprotected.

Using shared backup path protection (SBPP) [19] and multi-path approaches, to provide full protection against a single link failure, the backups have to provide the maximum bandwidth allocated on the links of the primary paths. In the resilient approach, when bandwidth is allocated to a request, we look for disjoint paths to be reserved as backup paths for that request. In practice, a request may not ask for full recovery of bandwidth demand upon occurrence of a failure and it is sufficient that a portion of the request demand is transmitted to the destination. Therefore, we compare the maximum primary allocation and the amount of requested backup and select the smaller value as the limit to be fulfilled by the backups. If this backup limit can not be found, it backtracks to the initial state and retries the bandwidth allocation with half of the primary bandwidth demand. This division by 2 is repeated until both primary and backup demands are satisfied. If the file can not be accommodated by its deadline, the scenario to which this request belongs, is rejected and all of its reservations have to be sent back to the network resource pool. Full details can be found in [2]. 


\subsection{Resilient AR Scheduling Architecture}

In this section, we briefly explain the heuristic-based resilient AR architecture, detailed in [2], for the reliable bandwidth scheduling problem. There are different blocks in this approach, presented in Fig. 1. As can be seen, new scenarios enter the scheduler through an API which can be transformed using the input transformation block. Then the resilient scheduling algorithm is invoked which follows a timeslot-based scheme. A timeslot is defined as a period of time in which reservations remain invariant. The new scenario is admitted and the schedule is updated provided that this algorithm succeeds in allocating bandwidth to all the scenario's requests. Otherwise, the previous scheduling remains unchanged.

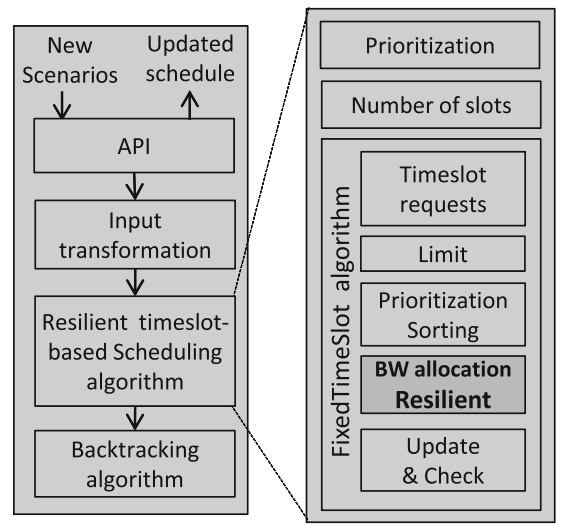

Fig. 1. Components of the resilient advance bandwidth reservation approach.

In the resilient scheduling component, first the requests within the scenario are prioritized and then the FixedTimeSlot algorithm is called. In the prioritization algorithm, the requests' priorities are assigned first based on the deadline, and then the request's demand. Requests with sooner deadline and higher demand receive higher priorities.

The FixedTimeSlot algorithm iterates over the time slots with 5 sub-modules. (1) TimeSlotRequests: determines the eligible requests which can be served at the current time slot. (2) Limit: defines a limitation for each request. For the streams this limit is equal to their demand which is fixed. For file-based requests the residual demand divided by timeslot size is considered as the limit. (3) Sorting: sorts the requests based on their priorities, assigned by the prioritization algorithm. (4) BWallocationResilient: responsible for resilient bandwidth allocation to the requests depending on their types. (5) Update and check for feasibility: Once the required demands are allocated to the requests, the schedule is updated if all the deadlines are met. Otherwise this schedule is infeasible. Full details can be found in [2]. 


\section{Optimized Resilient AR Scheduling}

In this section, we elaborate on how the reliable scheduling approach has been improved to achieve a higher request admittance ratio.

In the resilient approach, if the requested backup can not be found, it retries the primary allocation with fraction of the primary bandwidth demand $(50 \%$ in our case). Although this is a fast approach, we found that halving the request demand does not always lead to an optimal solution because we may miss the opportunity to transfer a higher volume of the file and the network capacity may not be fully utilized if other concurrent requests can not make use of it. As such, we propose to make better use of leftover capacities by deploying the binary search mechanism [20] for finding the maximum value which satisfies both primary allocation and the requested bandwidth demand. This way, perstep complexity of the reservations increases while a higher amount of allocations will potentially be achieved. We elaborate more on this with two examples.

Example 1: consider Fig. 2 with a file-based request of $300 \mathrm{~Gb}$ and timeslot size of $5 \mathrm{~min}$. The limit component sets $1 \mathrm{Gbps}(300 \mathrm{~Gb} / 300 \mathrm{~s})$ for the limitation of bandwidth reservations for this request. The resilient approach, first checks if it can fulfill both a 1 Gbps primary allocation and the requested backup demand. The amount of backup allocation depends on the percentage of requested protection and also the way the primary reservations are allocated. If the request's backup demand can not be fulfilled, the limit of the request is divided by 2 . Then the same procedure is repeated for the lower limit of $500 \mathrm{Mbps}$. If both $500 \mathrm{Mbps}$ primary demand and its corresponding backup are available, the primary and backup allocations are reserved and the request demand is updated by reducing reserved network capacity and the rest of the file has to wait for the next timeslots to be processed. Otherwise, this division by 2 is continued until the request limit is fulfilled or the file is finally rejected. However, this division by 2 is not efficient if the network is able to provide e.g. $400 \mathrm{Mbps}$. Based on this approach the resilient reservation approach can only provide $250 \mathrm{Mbps}$. This is shown in Fig. 2b following a multi-path allocation approach, i.e. $200+50 \mathrm{Mbps}$ for primary and $200 \mathrm{Mbps}$ (to cover single link failure) for shared backup paths. Although the $150 \mathrm{Mbps}$ can be occupied by other requests, this is not optimal and may results in a waste of network resources.

In order to improve the network utilization, we propose to make use of Binary Search to find an optimal value for the amount of reservations. This way if the algorithm recognizes that $500 \mathrm{Mbps}$ is not available but $250 \mathrm{Mbps}$ can be offered, instead of returning $250 \mathrm{Mbps}$, the algorithm tries to find the maximum available value between $250 \mathrm{Mbps}$ and $500 \mathrm{Mbps}$. The proposed solution first takes the middle value (375 Mbps) and checks the possibility of reservations again. As this is available, the algorithm again checks for the middle value between $375 \mathrm{Mbps}$ and $500 \mathrm{Mbps}$ which is $437.5 \mathrm{Mbps}$. This trend is continued with 406.25 and then 390.625 , etc. until the difference between the upper and lower bounds is smaller than a given margin, which we refer to as $\epsilon$. Assuming 2 Mbps as this margin the 


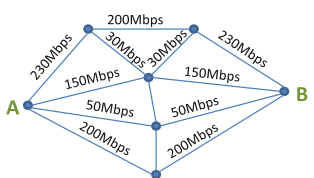

(a) Residual capacities

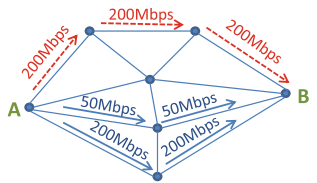

(b) Original approach

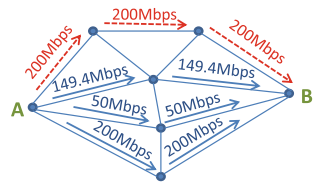

(c) Optimized approach

Fig. 2. An example of primary and backup reservations based on the original and the optimized resilient AR approaches. (Black: network capacity, Blue: Primary reservation, Red and dashed: Backup reservation) (Color figure online)

algorithm stops at 399.4 Mbps. The reservation based on the optimized resilient AR approach is shown in Fig. 2c. This margin can be altered to make a tradeoff between achieving a precise optimal value and solution complexity.

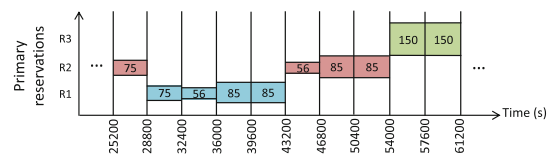

(a) Original resilient AR approach

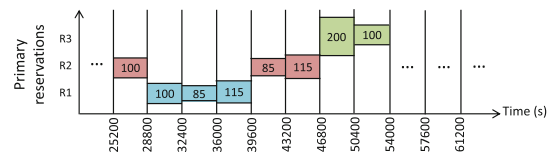

(b) Optimized resilient AR approach

Fig. 3. Comparing the primary allocations of the original and optimized versions of resilient timeslot-based advance bandwidth reservation approach.

Example 2: Figure 3 shows an example of a schedule for 3 file-based requests, $\mathrm{R} 1, \mathrm{R} 2$ and R3. The timeslot size is $1 \mathrm{~h}$ and in both figures only primary reservations are shown. In Fig. 3a and b, the reservations are made using the original and optimized version of the resilient advance bandwidth reservation approaches respectively. Figure $3 \mathrm{~b}$ reveals how the optimized resilient approach can improve network utilization and increase the probability of admittance for future requests. As can be seen, by allocating a higher volume of a given file, this file can be potentially transferred earlier compared to the original approach. This way, higher capacity is available for requests in future and the request admittance ratio will be potentially increased.

\section{Resilient Timeslot-Based AR Algorithms}

In this section, we elaborate on the optimized BWallocationResilient + algorithm, shown in Algorithm 1, which first assigns a cost to each network link using a cost allocation module. We have previously designed two algorithms for resilient bandwidth allocation depending on the type of the request, which we refereed to as BWallocationFBResilient and BWallocationVSResilient for filebased and streaming requests respectively. As we have optimized the resilient 
approach for file transfers, we do not elaborate on the BWallocationVSResilient algorithm. The common part for both algorithms is repeatedly finding the leastcost paths between source and destination of a given request until the limit of that request is fulfilled. However, provided that the limit of the request is not available, a different trend is followed by each approach. For the file-based request, maximum available capacity is reserved as the rest of the file can be processed during the next timeslots. For the streams, if there is not enough capacity to allocate to the request, it can not be served and thus the feasibility is set to false. The next step in the resilient algorithms is to find the backup paths.

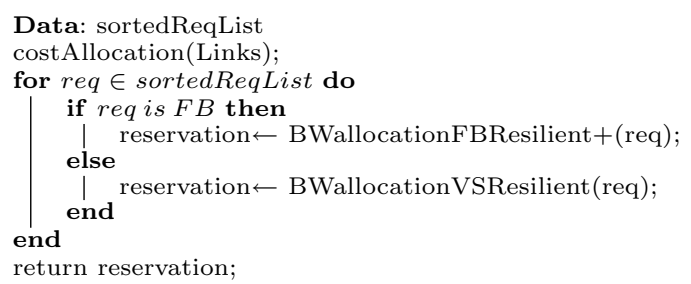

Algorithm 1: BWallocationResilient+

Depending on the backup demands and primary allocations, the amount of backup demand is first calculated. Both algorithms check if the backup can be fulfilled. In order to cover single failures, the backups have to be disjointed from the primary paths. As such, the links used in the primary paths are removed from the network and the bandwidth allocation algorithms are reused on the residual network to find the backup paths for that request. If the backups can be found, the primary and backup paths can be successfully allocated for the request. Otherwise, the primary paths have to be removed. Again if the backups for the streaming request are not fulfilled, the scheduling is not successful. However, for file transfers if the backup can not be provided, the algorithm tries with a lower primary bandwidth demand. This is repeated until both primary and backup demands of the file are satisfied. If this algorithm is being executed in the timeslot prior to the request deadline, and both primary and backup demands can not be fulfilled, the entire scenarios to which the file belongs, is rejected.

\subsection{BWallocationFBResilient+ Algorithm}

The main idea behind proposing the BWallocationFBResilient+ is to improve the performance of the $B$ WallocationFBResilient algorithm. In the BWallocationFBResilient algorithm, if the backup for a given request can not be provided, the limit of primary allocations is repeatedly halved and the possibility of the reservation is checked with this lower limit. We argue that this halving cycle can be improved by deploying a binary search algorithm. That is, given a file-based request, we seek for maximum available bandwidth which satisfies both primary and backup demands. Therefore, if the BWallocationFBResilient+ algorithm 


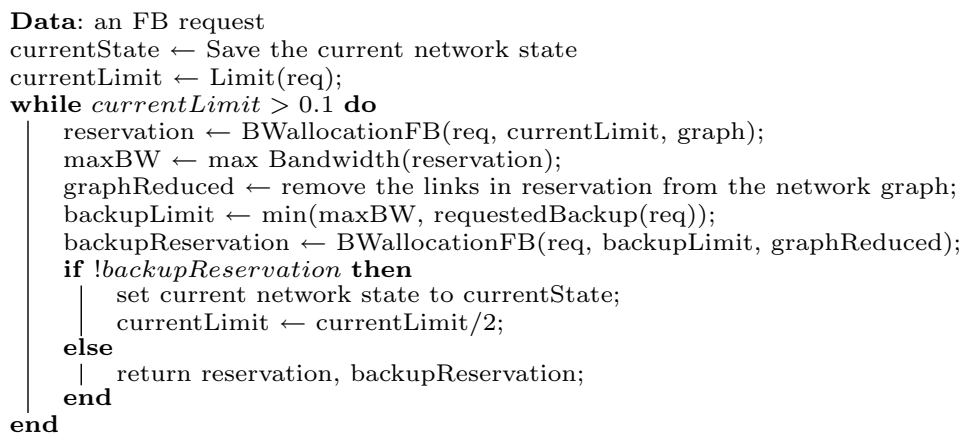

Algorithm 2: BWallocationFBResilient for file-based requests

finds that value $\mathrm{X}$ can satisfy both primary and backup demands, instead of returning this value, which was the case in the BWallocationFBResilient algorithm, a higher value based on the binary search approach is investigated and this is repeated until a near-optimal value (within an $\epsilon$ margin) is calculated and returned. This process is shown in Algorithm 3.

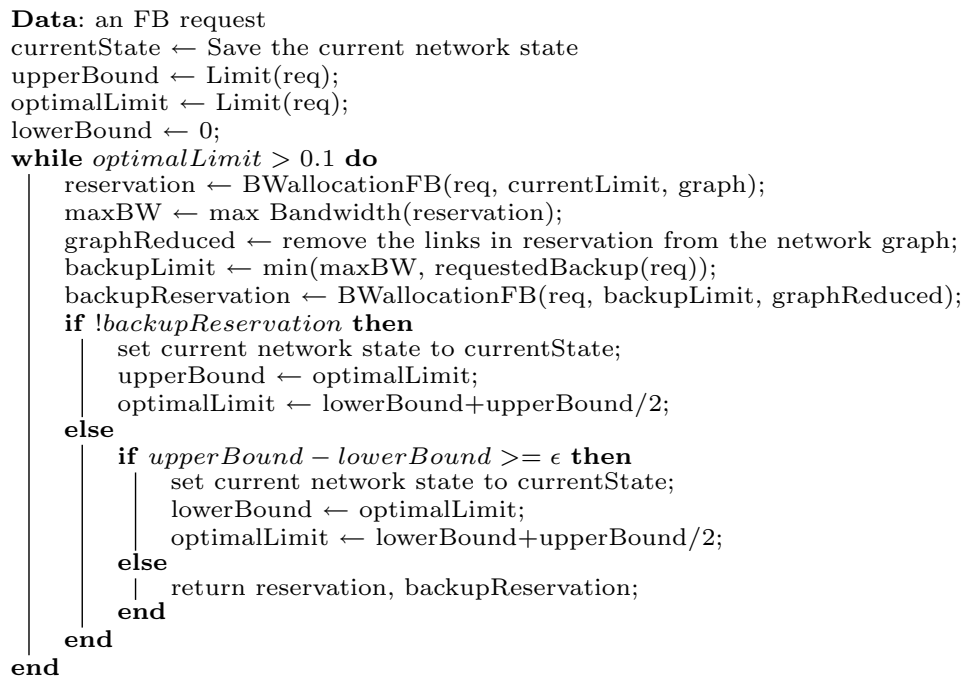

Algorithm 3: BWallocationFBResilient+ for file-based requests

\section{Performance Evaluation}

This section evaluates the quality and execution time of the proposed solution, compared to our previously designed resilient timeslot-based scheduling 
algorithms. For this analysis, SARA (Static Advance Reservation Approach) is evaluated in which all requests are known in advance, before the start of scheduling. The influence of the available network capacity, network load, backup demand, timeslot granularity and execution times are assessed.

\subsection{Evaluation Setup}

The network topology used for this evaluation contains 8 nodes and 16 bidirectional links. After discussion with our industrial partners in media production industry, 3 scenario types are defined: a soccer after-game discussion program, an infotainment show and a news broadcast program, consisting of 5, 18 and 8 interdependent file-based and video streaming requests respectively. A detailed overview of the randomized variables of requests and network topology can be observed from [1] and [21] respectively. In the fixed size timeslot-based solution, timeslot granularities of 5, 15 and 30 min and backup demand of $50 \%$ and 100\% are used.

Throughout this section, SARA[XX\%, YYmin] denotes that backup demand of $\mathrm{XX} \%$ and timeslot size of $Y Y$ minutes is considered in the fixed-size timeslotbased advance reservation algorithm. $S A R A+$ refers to the optimized resilient bandwidth reservation approach. In this approach the margin, which we referred to as $\epsilon$, equals $2 \mathrm{Mbps}$. Each simulation run covers a 24-h period. All results are averaged over 50 runs with different randomized inputs, error bars denote the standard error. All algorithms in this section are implemented in Java 8.

\subsection{Evaluation Results}

Evaluation of Network Capacity, Backup Demands and Timeslot Sizes: In Figs. 4 and 5, the network infrastructure has been configured for different available bandwidths, respectively for $100 \%$ and $50 \%$ backup demands to investigate the impact of available network capacity, backup demands and timeslot sizes on the performance of our algorithms in terms of percentage of admitted requests. In both evaluations, 7 scenarios (67 requests) are submitted to the bandwidth reservation system and the network capacities vary from $50 \mathrm{Mbps}$ to 400 Mbps.

What we can observe in these evaluations is as follows: first, the finer the timeslot size, the higher gain achieved by the $S A R A+$ approach. As can be observed from Fig. 4 , the $S A R A+$ approach is able to achieve on average up to $3.6 \%, 7.3 \%$ and $9.2 \%$ higher admittance ratio in 30-, 15- and 5-min timeslot sizes, respectively. Second, with higher backup demand, the performance of $S A R A+$ is more significant. In Fig. 5, with $50 \%$ backup demand, $S A R A+$ is able to outperform the $S A R A$ approach on average up to $8.5 \%$ with 5 -min timeslots. Comparing Figs. $4 \mathrm{c}$ and $5 \mathrm{c}$, the $S A R A+$ with $100 \%$ back up demand improves the request admittance ratio on average up to 2.8 times comparing to the $50 \%$ backup demand. 


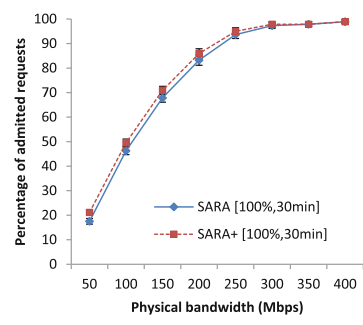

(a) 30-min timeslots

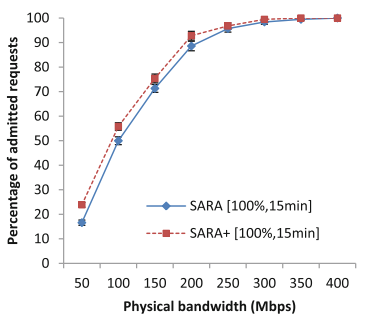

(b) 15-min timeslots

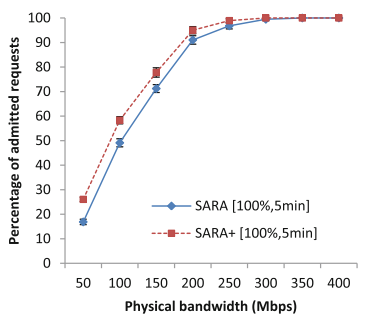

(c) 5-min timeslots

Fig. 4. Impact of timeslot size with 100\% backup demand in the timeslot-based advance bandwidth reservation approach.

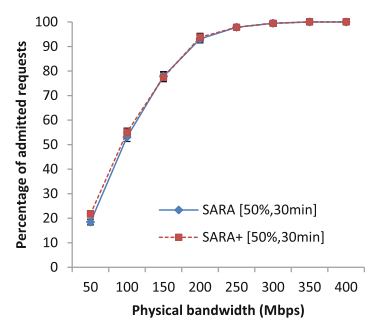

(a) 30-min timeslots

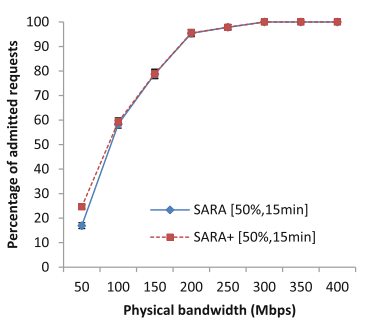

(b) 15-min timeslots

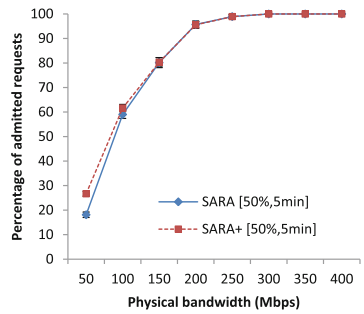

(c) 5-min timeslots

Fig. 5. Impact of timeslot size with $50 \%$ backup demand in the timeslot-based advance bandwidth reservation approach.

\section{Evaluation of Network Load, Timeslot Sizes and Execution Times:} Figures 6 and 7 compare the influence of the network load and timeslot sizes on the quality and time complexity of our algorithms. Backup demand of $100 \%$ and network capacity of $200 \mathrm{Mbps}$ are used.

As can be seen in Fig. 6, by increasing the number of scenarios, the percentage of admitted requests decreases and the $S A R A+$ approach performs better with fine-grained timeslot sizes. We notice that the advance bandwidth reservation system gains more by deploying the $S A R A+$ approach and with the 5 -min timeslot size, shows up to $7.3 \%$ higher request admittance ratio.

The time complexity of the approaches are evaluated in Fig. 7 for an increasing range of scenarios. This figure reveals that the granularity of timeslot size impacts the execution times of both approaches differently. While with 30-min timeslot size, the execution time of $S A R A+$ is up to 147 milliseconds higher compared to the $S A R A$ approach, with 5 -min timeslots, this time is up to 4.5 second lower. These results indicate that the quality and complexity of the advance bandwidth reservation system can be improved by deploying the $S A R A+$ approach with fine-grained timeslot sizes.

For further investigation of the execution time, we have assessed the impact of network capacity on the execution time, when the timeslot granularity of $5 \mathrm{~min}$ is used. This has been shown in Fig. 8. The number of scenarios is 7 and 14 


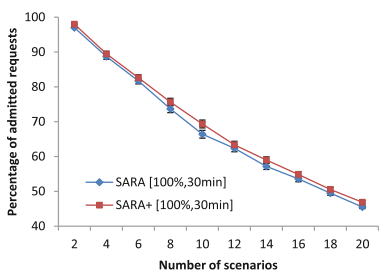

(a) 30-min timeslots

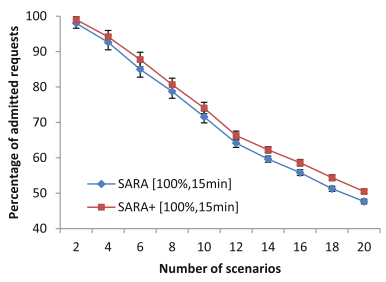

(b) 15-min timeslots

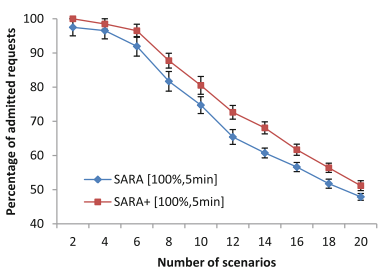

(c) 5-min timeslots

Fig. 6. Impact of network load in the fixed size timeslot-based advance bandwidth reservation approach.

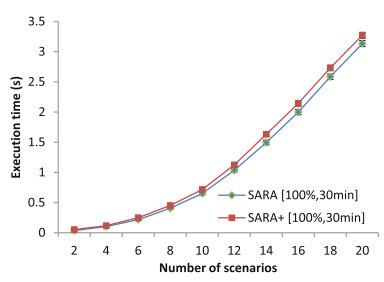

(a) 30-min timeslots

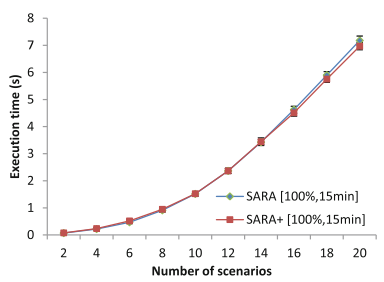

(b) 15-min timeslots

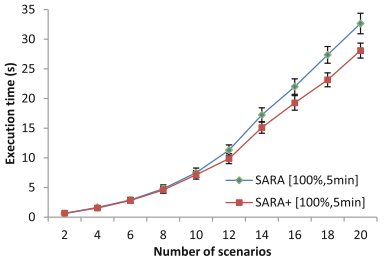

(c) 5-min timeslots

Fig. 7. Comparing the execution times in the fixed size timeslot-based advance bandwidth reservation approach.

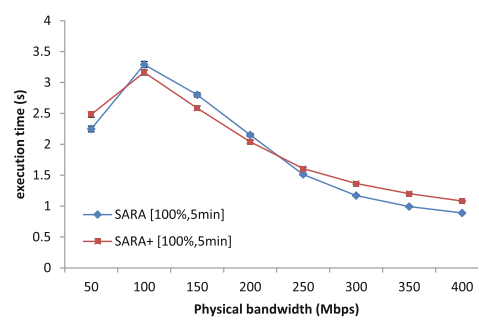

(a) 7 scenarios

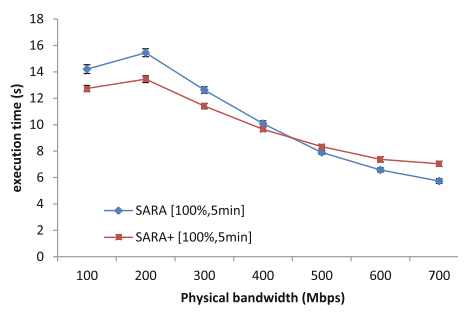

(b) 14 scenarios

Fig. 8. Comparing the execution times in the function of network capacity in the fixed size timeslot-based advance bandwidth reservation approach.

in Fig. 8a and b respectively. This evaluation shows that when there is enough bandwidth capacity available, the $S A R A$ approach is able to perform faster while $S A R A+$ can better manage the time under stressed network conditions, i.e. limited network capacity.

\section{Conclusions}

In this paper, we have optimized the resilient scheduling algorithms, previously presented for advance bandwidth reservation in media-centric networks. In the 
original version, for a given file transfer, if both primary and backup demands can not be fulfilled, the algorithm is repeatedly executed with $50 \%$ of primary demand until both demands are fulfilled or the request is rejected. We proposed to make use of binary search instead of halving the primary demand and showed that this optimization improves the performance of the timeslot-based advance reservation system in terms of request admittance ratio. The impact of available capacity, network load, timeslot sizes and backup demands is evaluated. Based on the results, we can conclude that the proposed solution specifically performs well under limited network capacity and with fine-grained timeslot sizes. The proposed approach outperform the original one both in terms of the execution time, with 5-min timeslot size, and the percentage of admitted requests, up to $9.2 \%$.

Acknowledgment. This work has been performed within the context of ICON MECaNO, a project co-funded by iMinds, a digital research institute founded by the Flemish Government. Project partners are SDNsquare, Limecraft, VideoHouse, Alcatel-Lucent and VRT, with project support from IWT under grant agreement no. 130646 .

\section{References}

1. Barshan, M., Moens, H., Famaey, J., De Turck, F.: Deadline-aware advance reservation scheduling algorithms for media production networks. Comput. Commun. 77, 26-40 (2016)

2. Sahhaf, S., Barshan, M., Tavernier, W., Moens, H., Colle, D., Pickavet, M.: Resilient algorithms for advance bandwidth reservation in media production networks. In: International Conference on the Design of Reliable Communication Networks (DRCN), pp. 130-137. IEEE (2016)

3. Charbonneau, N., Vokkarane, V.M.: A survey of advance reservation routing and wavelength assignment in wavelength-routed WDM networks. IEEE Commun. Surv. Tutor. 14(4), 1037-1064 (2012)

4. Burchard, L.-O., Heiss, H.-U., De Rose, C.: Performance issues of bandwidth reservations for grid computing. In: Symposium on Computer Architecture and High Performance Computing, pp. 82-90 (2003)

5. Naikstam, S., Figueira, S.: Elastic reservations for efficient bandwidth utilization in lambdagrids. Fut. Gener. Comput. Syst. 23(1), 1-22 (2007)

6. Rajah, K., Ranka, S., Xia, Y.: Advance reservations and scheduling for bulk transfers in research networks. IEEE Trans. Parallel Distrib. Syst. 20, 1682-1697 (2009)

7. Xie, C., Alazemi, H., Ghani, N.: Rerouting in advance reservation networks. Comput. Commun. 35(12), 1411-1421 (2012)

8. Zuo, L., Zhu, M.M., Wu, C.Q.: Fast and efficient bandwidth reservation algorithms for dynamic network provisioning. J. Netw. Syst. Manag. (2013)

9. Alazemi, H., Xu, F., Xie, C., Ghani, N.: Advance reservation in distributed multidomain networks. IEEE Syst. J. (2013)

10. Guok, C., Engineer, E.N., Robertson, D.: Esnet on-demand secure circuits and advance reservation system (oscars). Internet2 Joint (2006) 
11. Gibbard, B., Katramatos, D., Yu, D.: Terapaths: end-to-end network path qos configuration using cross-domain reservation negotiation. In: 3rd International Conference on Broadband Communications, Networks and Systems (BROADNETS), pp. 1-9. IEEE (2006)

12. Gu, J., Katramatos, D., Liu, X., Natarajan, V., Shoshani, A., Sim, A., Yu, D., Bradley, S., McKee, S.: Stornet: integrated dynamic storage and network resource provisioning and management for automated data transfers. J. Phys.: Conf. Ser. 331, 012002 (2011). IOP Publishing

13. Sharma, S., Katramatos, D., Yu, D., Shi, L.: Design and implementation of an intelligent end-to-end network qos system. In: Proceedings of the International Conference on High Performance Computing, Networking, Storage and Analysis, SC 2012, USA, pp. 68:1-68:11. IEEE Computer Society Press (2012)

14. Watanabe, T., Omizo, T., Akiyama, T., Iida, K.: Resilientflow: deployments of distributed control channel maintenance modules to recover SDN from unexpected failures. In: 11th International Conference on the Design of Reliable Communication Networks (DRCN), pp. 211-218. IEEE (2015)

15. Tanwir, S., Battestilli, L., Perros, H., Karmous-Edwards, G.: Dynamic scheduling of network resources with advance reservations in optical grids. Int. J. Netw. Manag. 18(2), 79-106 (2008)

16. Li, T., Wang, B., Xin, C., Zhang, X.: On survivable service provisioning in WDM optical networks under a scheduled traffic model. In: IEEE Global Telecommunications Conference, GLOBECOM 2005, vol. 4, p. 5-pp. IEEE (2005)

17. Li, T., Wang, B.: On optimal survivability design in WDM optical networks under a scheduled traffic model. In: 5th International Workshop on Design of Reliable Communication Networks (DRCN), p. 8-pp. IEEE (2005)

18. Cavdar, C., Tornatore, M., Buzluca, F., Mukherjee, B.: Dynamic scheduling of survivable connections with delay tolerance in WDM networks. In: IEEE INFOCOM Workshops 2009, pp. 1-6. IEEE (2009)

19. Cavdar, C., Tornatore, M., Buzluca, F., Mukherjee, B.: Shared-path protection with delay tolerance (SDT) in optical WDM mesh networks. J. Lightwave Technol. 28(14), 2068-2076 (2010)

20. Lehmer, D.H.: Teaching combinatorial tricks to a computer. In: Proceedings of Symposium in Applied Mathematics Combinatorial Analysis, vol. 10, pp. 179-193 (1960)

21. Barshan, M., Moens, H., Volckaert, B.: Dynamic adaptive advance bandwidth reservation in media production networks. In: IEEE NetSoft Conference and Workshops (NetSoft), pp. 58-62. IEEE (2016) 
Open Access This chapter is licensed under the terms of the Creative Commons Attribution 4.0 International License (http://creativecommons.org/licenses/by/4.0/), which permits use, sharing, adaptation, distribution and reproduction in any medium or format, as long as you give appropriate credit to the original author(s) and the source, provide a link to the Creative Commons license and indicate if changes were made.

The images or other third party material in this chapter are included in the chapter's Creative Commons license, unless indicated otherwise in a credit line to the material. If material is not included in the chapter's Creative Commons license and your intended use is not permitted by statutory regulation or exceeds the permitted use, you will need to obtain permission directly from the copyright holder.

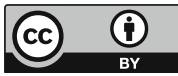

